\title{
Regulatory region mutations of TERT, PLEKHSI and GPR 126 genes as urinary biomarkers in upper tract urothelial carcinomas
}

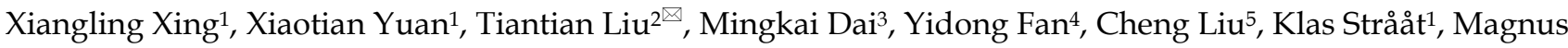 \\ Björkholm ${ }^{1}$, Dawei $\mathrm{Xu}^{1}$ \\ 1. Department of Medicine, Division of Hematology, Bioclinicum and Center for Molecular Medicine (CMM), Karolinsk Institutet and Karolinska University Hospital \\ Solna, SE-17176 Stockholm, Sweden. \\ 2. Pathology Department, School of Basic Medical Science, Shandong University, Jinan, PR China. \\ 3. Central Research Laboratory, the Second Hospital, Cheeloo College of Medicine, Shandong University, Jinan, 250033, PR China. \\ 4. Department of Urology, Qilu Hospital, Cheeloo College of Medicine, Shandong University, Jinan, 250012, PR China. \\ 5. Department of Urology, Peking University Third Hospital, Beijing, PR China. \\ $\triangle$ Corresponding author: Dr. Tiantian Liu, Pathology Department, School of Basic Medical Science, Shandong University, Jinan, PR China. Phone: +86 18205419616; E-mail: \\ liu.tiantian@sdu.edu.cn. \\ (c) The author(s). This is an open access article distributed under the terms of the Creative Commons Attribution License (https://creativecommons.org/licenses/by/4.0/). \\ See http://ivyspring.com/terms for full terms and conditions.
}

Received: 2020.12.04; Accepted: 2021.04.22; Published: 2021.05.05

\begin{abstract}
Background: The hotspot regulatory region mutations of the TERT, PLEKHSI and GPR 126 genes have been shown to occur frequently in urothelial bladder carcinoma (UBC). However, it is currently unclear whether these mutations are all present in upper tract urothelial carcinomas (UTUC) including renal pelvic carcinoma (RPC) and ureter carcinoma (UC), although TERT promoter mutations were previously observed in these malignancies.

Methods: The hotspot mutations of TERT and PLEKHSI promoters and GPR126 intron 6 (enhancer) in tumors derived from 164 patients with UTUC were determined using Sanger sequencing, and the obtained results were further compared with the mutation frequency in 106 UBCs. The mutations were also assessed in urine from patients with UTUC and UBC.

Results: The mutation frequencies in UTUC tumors were $28 \%, 5.8 \%$ and $11 \%$ for TERT and PLEKHS1 promoters and GPR126 intron 6, respectively, which were lower than those $(44.3 \%, 26.4 \%$, and $31.4 \%$, respectively) in UBCs. The total frequencies for the presence of any of these mutations were $50.8 \%$ and $34.4 \%$ for RPCs and UCs, respectively. All these mutated DNA sequences were detectable in urine from both UTUC and UBC patients and disappeared rapidly in most patients after surgery.

Conclusions: This proof-of-concept study demonstrates that the hotspot mutations in the TERT, PLEKHS1 and GPR 126 non-coding regions are present in UTUCs, and that urinary assays of these mutated sequences serve as potential biomarkers for UTUC diagnostics and disease monitoring.
\end{abstract}

Key words: GPR126; PLEKHS1; TERT; Urinary biomarker; UBC; UTUC

\section{Introduction}

Urothelial carcinomas originate from the urothelium or belong to transitional cell carcinomas, and consist primarily of upper tract urothelial carcinomas (UTUCs) and urothelial bladder carcinomas (UBCs) [1]. UTUCs include renal pelvic carcinomas (RPCs) and ureter carcinomas (UCs), and their incidence is much lower compared to UBCs, but has increased gradually over the past two decades, especially in East Asia including China [1, 2]. The majority of UTUCs have become invasive or metastatic when diagnosed, mainly due to the lack of early clinical symptoms and of specific diagnostic approaches [3-7]. Currently, UTUC diagnosis is mainly based on imaging and endoscopy, while non-invasive methods such as bladder urine cytology, a standard tool for UBC diagnostics, has very limited sensitivity in UTUCs [5, 7]. In order to improve clinical management and outcomes of UTUCs, oncologists and scientists are striving to search for 
reliable disease bio-markers and to develop accurate diagnostic tools.

The activation of telomerase, a RNA-dependent DNA polymerase lengthening telomeres, is essential to the malignant transformation of human cells $[8,9]$. Recently, the recurrent mutation in the proximal promoter of the telomerase reverse transcriptase (TERT) gene, which encodes the catalytic, rate-limiting component of the telomerase complex, has been identified to drive telomerase activation in many human malignancies including UTUCs and UBCs [10-15]. Two hotspot mutations in the TERT promoter so-called C228T and C250T create de novo ETS binding motifs, thereby activating TERT transcription and telomerase $[16,17]$. We previously observed that the frequencies of TERT promoter mutations were $48 \%$ and $19 \%$ in primary RPC and UC tumors, respectively [12]. Moreover, the mutated TERT promoter DNA was detectable in patients' urine, and thus served as a urine-based diagnostic marker [12]. However, as described above, more than $50 \%$ of RPC and $80 \%$ UC tumors carry a wild type (wt) TERT promoter, and hence, other urinary biomarkers are required for these patients.

Recent studies have identified the hotspot mutations in the PLEKHS1 promoter and GPR126 intron 6 in UBCs [18-20]. In UBC primary tumors, mutation rates were approximately $30-48 \%$ and $50 \%$ for the PLEKHS1 promoter and GPR126 intron 6, respectively $[19,20]$. Both UTUCs and UBCs originate from the urothelium and share certain molecular events in the pathogenesis, however, their molecular alterations may be distinct, or differ substantially due to different anatomical locations or other unknown reasons [1, 4]. It is currently unclear whether these mutations also occur in RPCs and UCs, and if so, whether they overlap with TERT promoter mutations, or whether they can be used as biomarkers in patients with a wt TERT promoter. In the present study, we simultaneously determined the mutations in the TERT and PLEKHS1 promoters and GPR126 intron 6 in UTUC tumors, compared the results with UBCs, and finally evaluated their potential clinical implications.

\section{Patients and methods}

\section{UTUC and UBC patients and tumor specimens}

The study was conducted on 164 patients with UTUC (68 RPCs and 96 UCs) and 106 patients with UBC who underwent surgery at Shandong University Qilu Hospital and Second Hospital, China. UTUC and UBC were diagnosed according to the criteria of the World Health Organization (WHO) [21]. Patients' characteristics are summarized in Tables 1 and 2, which include sex, age at diagnosis, tumor size, pathology stage, clinical stage/metastases. The specimens were collected after surgical treatment and kept frozen at $-80{ }^{\circ} \mathrm{C}$. All samples were collected with written informed consent and the study was approved by the Shandong University Second Hospital ethics committee.

\section{Voided urine samples from UTUC and UBC patients}

Spontaneously voided urine was collected from 89 patients including 13 UTUCs and 76 UBCs prior to surgical treatment. In 20 of these 89 patients, urine was also consecutively obtained one week postoperation. Fifty $\mathrm{ml}$ of urine were centrifuged and cell pellets were kept at $-80^{\circ} \mathrm{C}$ until use.

\section{DNA extraction and sequencing}

Genomic DNA was extracted from frozen tumor tissue samples and urine pellets using QIAGEN DNA extraction kits. The mutations of TERT and PLEKHS1 promoters and GPR126 intron 6 were analyzed using Sanger sequencing as described $[22,23]$. The following primers were used for Sanger sequencing: The TERT promoter: 5'-CAC CCG TCC TGC CCC TTC ACC TT-3' (forward) and 5'-GGC TTC CCA CGT GCG CAG CAG GA-3' (reverse); the PLEKHS1 promoter: 5'-GAA TCC TCG GGA CAA GGC ACT-3' (forward) and 5'-GTC AGT CCT ATT TCC CTC TGA CT-3' (reverse); GPR126 intron 6: 5'-CCA AGG AGA TTT ATG ATG GAG CAA-3' (forward) and 5'-GCA GAG AGA GAT GGC CTA AAC A-3' (reverse). The mutations were verified by sequencing from both directions.

\section{Statistical analyses}

Unless stated, all statistical analyses were performed using IBM SPSS Statistics version 24 (IBM, Armonk, NY). Differences in the TERT and PLEKHS1 promoter or GPR126 intron 6 mutation frequencies in relation to sex, TNM stage, pathology stage, tumor size, number of tumors, recurrence, were determined using Chi-Square or Fisher's Exact Test. MannWhitney $U$ Test was used to analyze differences in age between the mutation-positive $(\mathrm{mt})$ and negative $(\mathrm{wt})$ groups. $\mathrm{P}$ values of $<0.05$ were considered as statistically significant. Sensitivity is defined as numbers of true mutation-positive samples/numbers of mutation positive samples detected from tumor tissues. Specificity is defined as numbers of true mutation-negative samples/numbers of mutationnegative samples in tumor tissues. Accuracy is defined as numbers of correct assessments/numbers of all samples.

To visualise proportions of different mutations 
in both tumor and urine samples, annotated pie chart and permutation test was performed using SPICE 6.0 (NIH). Patient samples with any missing mutation information were excluded from the SPICE analysis.

\section{Results}

\section{The mutation frequency of TERT and PLEKHS1 promoters and GPR126 intron 6 in UTUC tumors}

Mutational status in TERT and PLEKHS1 promoters and GPR126 intron 6 was analyzed in tumor DNA derived from 164 patients with UTUC using Sanger sequencing (Fig. 1). A total of 164 patients in the present cohort were evaluable for the TERT promoter status: the tumors carrying a wt and mutated (mt) TERT promoter were $118(72 \%)$ and 46 (28\%), respectively (Fig. 1A). The C228T mutation was predominant $(37 / 46,80 \%)$ while C250T occurred in only $9 / 46(20 \%)$ of tumors (Table 1$)$. For the PLEKHS1 promoter and GPR126 intron sequencing, 155 of 164 tumors were successfully studied (Table 1). Nine of 155 tumors (5.8\%) harboured the $\mathrm{mt}$ PLEKHS1 promoter, among which $6(6 / 64,9.4 \%)$ were RPCs while $3(3 / 91,3.3 \%)$ were UCs (Fig. 1B) (Tables 2 and 3). In these tumors bearing the mt PLEKHS1 promoter, 6/9 had $C>T$, while 3 of them were $G>A$ mutations. The GPR intron 6 mutation was identified in 17 of 155 tumors (11\%), which included 5 RPCs (5/62, 8.1\%) and 12 UCs (12/93, 12.9\%) (Tables 2 and $3)$. These results thus demonstrate that all these mutations occur in UTUCs with different frequencies.

The mutations of the TERT and PLEKHS1 promoters and GPR126 intron 6 occurred in most UTUC tumors separately whereby only less than 3\% of the cohort had co-existing mutations (Fig. 2), and there was no association between these three mutations. In a small fraction of tumors, TERT promoter mutations co-existed with either PLEKHS1 promoter (3 tumors) or GPR126 intron 6 (4 tumors) mutations, whereas the PLEKHS1 promoter and GPR126 mutations occurred together in 2 UTUC tumors. The total frequencies for the presence of any of these mutations were $50.8 \%$ and $34.4 \%$ for RPCs and UCs, respectively.

\section{Comparison of mutation frequencies between UTUCs and UBCs}

The data above indicate much lower frequencies in PLEKHS1 and GPR126 mutations in UTUCs than observed in UBCs, as recently reported [18-20]. However, such a difference may be also due to the differences in ethnicity, geography or environment and carcinogen exposure [11]. To elucidate this question, we further analyzed 106 UBC patients diagnosed and treated at the same hospitals, both cohorts of UBC and UTUC patients being Han Chinese. The TERT and PLEKHS1 promoter and GPR126 intron 6 mutations in this cohort of UBC patients were $47 / 106(44.3 \%), 28 / 106(26.4 \%)$, and $32 / 102(31.4 \%)$, respectively, which were indeed significantly higher than those in the present cohort of UTUCs (Fig. 2 and Table 4). The total frequencies for the presence of any of these mutations were $67.9 \%$, much higher than that in UTUCs. Five UBC tumors carried all three mutations, while the co-occurrence of the $\mathrm{mt}$ TERT promoter together with the mt PLEKHS1 promoter and GPR126 intron 6 were found in 6 and 13 UBC tumors, respectively.

\section{Detection of TERT and PLEKHSI promoter and GPR 126 intron 6 mutations in urine from patients with UTUC and UBC}

We and others previously showed that the mutated TERT promoter sequence was detectable in urine derived from UTUC and UBC patients, and served as a urinary diagnostic biomarker [10-12, 15, 24]. Here we further sought to determine whether all these mutated fragments could be found in the urine from UTUC and UBC patients. The urinary samples from 13 UTUC patients and 76 UBC patients were collected before surgery and Sanger sequencing was carried out. As shown in Table 5, the mutated TERT and PLEKHS1 promoters and GPR126 intron 6 were indeed detected in patient urine, although the sensitivity for Sanger sequencing was only $60 \%$. Nonetheless, a high consistency between tumors and urine reflects satisfactory specificity (Table 5). In addition, the urinary samples collected one week post-operation surgery were available in 20 of these patients and 10 of them were positive for mutated TERT and/or PLEKHS1 promoters or GPR126 intron 6 in their urine before surgery. In general, we observed a significant reduction in the frequencies of mutated fragments from post-operative samples (Fig. 3). The sequencing analysis showed that the mutated DNA fragments in urine disappeared in 6 of 10 patients after tumor removal. Their rapid disappearance indicates that they may be also reliable biomarkers for disease surveillance of UTUCs and UBCs.

\section{Association of the mutations with clinical variables in UTUCs and UBCs}

We finally determined a potential relationship between the detected mutations and additional clinical variables in patients with UTUC and UBC (Tables 1 and 4). For TERT promoter mutations, a significantly higher frequency was seen in older UC patients $(P=0.005)$, but there were no associations 
between the TERT promoter mutation and TNM or pathological stages, tumor sizes, metastasis or recurrence in UC or RPC (Tables 2 and 3). In UBCs, the presence of mutated TERT promoter mutations was significantly associated with disease recurrence and male sex $(P=0.021$ and 0.041 , respectively) (Table
4). For PLEKHS1 promoter and GPR126 intron 6 mutations, no associations with any of tested clinic-pathological variables were observed in UTUCs (Table 2 and 3), while the GPR126 mutations were associated with small tumors in patients with UBCs (< $3 \mathrm{~cm}$ vs $\geq 3 \mathrm{~cm}, P=0.006$ ) (Table 4 ).

Table 1. Clinic-pathological variables and association with TERT and PLEKHSI promoter and GPR 126 intron 6 mutations in UTUC patients

\begin{tabular}{|c|c|c|c|c|c|c|c|c|c|}
\hline & \multicolumn{3}{|c|}{ TERT promoter $(\mathrm{N}=164)$} & \multicolumn{3}{|c|}{ PLEKHS1 promoter $(\mathrm{N}=155)$} & \multicolumn{3}{|c|}{ GPR126 intron $6(\mathrm{~N}=155)$} \\
\hline & $\mathrm{wt}$ & $\mathrm{mt}$ & $P$ & $\mathrm{wt}$ & $\mathrm{mt}$ & $P$ & wt & $\mathrm{mt}$ & $P$ \\
\hline & $118 / 164(72 \%)$ & $46 / 164(28 \%)$ & & $146 / 155(94.2 \%)$ & $9 / 155(5.8 \%)$ & & $138 / 155(89 \%)$ & $17 / 155(11 \%)$ & \\
\hline Age (Median, years) & $67(32-83)$ & $65(36-87)$ & 0.643 & $67(36-87)$ & $72(58-78)$ & 0.157 & $65(36-87)$ & $68(32-81)$ & 0.337 \\
\hline Gender & & & 0.356 & & & 1.000 & & & 0.351 \\
\hline Female & 45 & 14 & & 50 & 3 & & 49 & 8 & \\
\hline Male & 73 & 32 & & 96 & 6 & & 89 & 9 & \\
\hline TNM stage & & & 0.429 & & & 0.685 & & & 0.765 \\
\hline $\mathrm{pTa}+\mathrm{pT} 1$ & 30 & 9 & & 34 & 1 & & 35 & 3 & \\
\hline$\geq \mathrm{pT} 2$ & 88 & 37 & & 112 & 8 & & 103 & 14 & \\
\hline Pathology stage & & & 0.664 & & & 0.135 & & & 0.549 \\
\hline G1 & 19 & 9 & & 27 & 0 & & 24 & 1 & \\
\hline G2 & 9 & 5 & & 12 & 2 & & 12 & 1 & \\
\hline G3 & 90 & 32 & & 107 & 7 & & 102 & 15 & \\
\hline Tumor size & & & 0.731 & & & 0.077 & & & 0.743 \\
\hline$<3 \mathrm{~cm}$ & 46 & 18 & & 58 & 1 & & 54 & 7 & \\
\hline$\geq 3 \mathrm{~cm}$ & 54 & 24 & & 68 & 8 & & 65 & 7 & \\
\hline LN metastasis & & & 0.285 & & & 1.000 & & & 1.000 \\
\hline No & 109 & 45 & & 137 & 9 & & 130 & 16 & \\
\hline Yes & 9 & 1 & & 9 & 0 & & 8 & 1 & \\
\hline Distant metastasis & & & 0.135 & & & 0.262 & & & 0.445 \\
\hline No & 116 & 43 & & 142 & 8 & & 134 & 16 & \\
\hline Yes & 2 & 3 & & 4 & 1 & & 4 & 1 & \\
\hline Recurrence & & & 0.313 & & & 1.000 & & & 0.375 \\
\hline No & 116 & 44 & & 142 & 9 & & 135 & 16 & \\
\hline Yes & 2 & 2 & & 4 & 0 & & 3 & 1 & \\
\hline
\end{tabular}

LN: Lymph node; mt: mutant; wt: wild type; UTUC: Upper track urothelial carcinoma.

Table 2. Clinic-pathological variables and association with TERT and PLEKHSI promoter and GPR 126 intron 6 mutations in RPC patients

\begin{tabular}{|c|c|c|c|c|c|c|c|c|c|}
\hline & \multicolumn{3}{|c|}{ TERT promoter $(\mathrm{N}=68)$} & \multicolumn{3}{|c|}{ PLEKHS1 promoter $(\mathrm{N}=64)$} & \multicolumn{3}{|c|}{ GPR126 intron $6(\mathrm{~N}=62)$} \\
\hline & $\overline{w t}$ & $\mathrm{mt}$ & $P$ & wt & $\mathrm{mt}$ & $P$ & wt & $\mathrm{mt}$ & $P$ \\
\hline & $41 / 68(60.3 \%)$ & $27 / 68(39.7 \%)$ & & $58 / 64(90.6 \%)$ & $6 / 64(9.4 \%)$ & & $57 / 62(91.9 \%)$ & $5 / 62(8.1 \%)$ & \\
\hline Age (Median, years) & $67(40-80)$ & $64(36-77)$ & 0.156 & $63(36-80)$ & $72.5(58-78)$ & 0.095 & $63(36-80)$ & $69(64-73)$ & 0.174 \\
\hline Gender & & & 0.286 & & & 0.671 & & & 1.000 \\
\hline Female & 19 & 9 & & 22 & 3 & & 24 & 2 & \\
\hline Male & 22 & 18 & & 36 & 3 & & 33 & 3 & \\
\hline TNM stage & & & 0.787 & & & 0.584 & & & 0.574 \\
\hline pTa + pT1 & 8 & 6 & & 12 & 0 & & 13 & 0 & \\
\hline$\geq \mathrm{pT} 2$ & 33 & 21 & & 46 & 6 & & 44 & 5 & \\
\hline Pathology stage & & & 0.487 & & & 0.312 & & & 0.699 \\
\hline G1 & 7 & 6 & & 12 & 0 & & 10 & 0 & \\
\hline G2 & 2 & 3 & & 4 & 1 & & 4 & 0 & \\
\hline G3 & 32 & 18 & & 42 & 5 & & 43 & 5 & \\
\hline Tumor size & & & 0.140 & & & 0.176 & & & 0.648 \\
\hline$<3 \mathrm{~cm}$ & 10 & 11 & & 17 & 0 & & 17 & 2 & \\
\hline$\geq 3 \mathrm{~cm}$ & 30 & 15 & & 39 & 6 & & 38 & 3 & \\
\hline LN metastasis & & & 0.514 & & & 1.000 & & & 1.000 \\
\hline No & 39 & 27 & & 56 & 6 & & 55 & 5 & \\
\hline Yes & 2 & 0 & & 2 & 0 & & 2 & 0 & \\
\hline Distant metastasis & & & 0.154 & & & 0.180 & & & 1.000 \\
\hline No & 41 & 25 & & 57 & 5 & & 55 & 5 & \\
\hline Yes & 0 & 2 & & 1 & 1 & & 2 & 0 & \\
\hline
\end{tabular}

LN: Lymph node; mt: mutant; wt: wild type; RPC: Renal pelvic carcinoma. 
$\begin{array}{ccc}1295135 & 1295113 \\ \text { A cecgacecetcecgggtececggeccagececetceggg }\end{array}$

$\underset{(\mathrm{C} 250 \mathrm{~T})}{\downarrow} \underset{\text { binding motif }}{\stackrel{\text { ETS }}{\downarrow} \underset{(\mathrm{C} 228 \mathrm{~T})}{\stackrel{\boldsymbol{c c t t}}{\downarrow}}} \rightarrow$
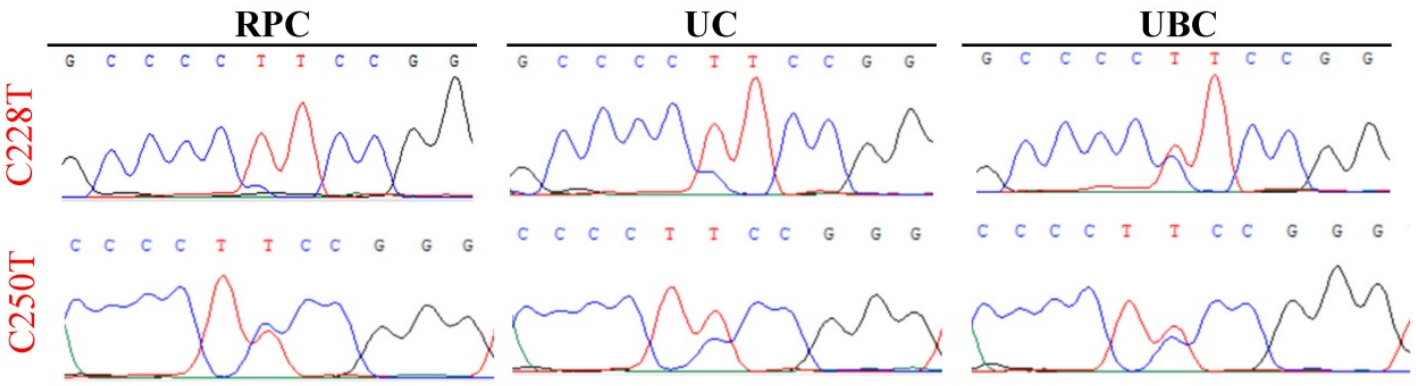

B

$113751831 \quad 113751834$

Chromosome 10

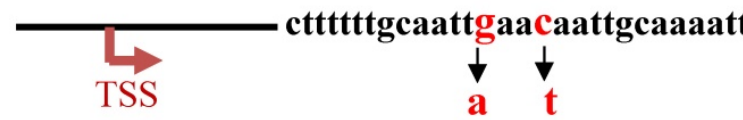

RPC

T T T T G C-A A T T A A A C-A A T T G C A A-A A

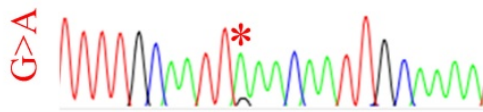

C TTG C A A T-TG A-ATA A TTG C-A A A:
UC

T T T G CAAT TA AA CA A T T G C-A

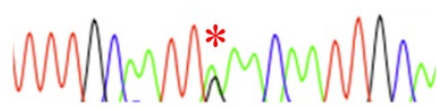

ГT T T G C-A A T T G A A T-A A T T G C A A

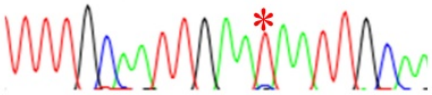

Chromosome 5

TERT promoter
TSS ATG

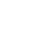


Table 3. Clinic-pathological variables and association with TERT and PLEKHSI promoter and GPR 126 intron 6 mutations in UC patients

\begin{tabular}{|c|c|c|c|c|c|c|c|c|c|}
\hline & \multicolumn{3}{|c|}{ TERT promoter $(\mathrm{N}=96)$} & \multicolumn{3}{|c|}{ PLEKHS1 promoter $(\mathrm{N}=91)$} & \multicolumn{3}{|c|}{ GPR126 intron $6(\mathrm{~N}=93)$} \\
\hline & wt & $\mathrm{mt}$ & $P$ & wt & $\mathrm{mt}$ & $P$ & wt & $\mathrm{mt}$ & $P$ \\
\hline & $77 / 96(80.2 \%)$ & $19 / 96(19.8 \%)$ & & $88 / 91(96.7 \%)$ & $3 / 91(3.3 \%)$ & & $81 / 93(87.1 \%)$ & $12 / 93(12.9 \%)$ & \\
\hline Age (Median, years) & $67(32-83)$ & $74(55-87)$ & 0.005 & $67.5(41-87)$ & $71(64-76)$ & 0.519 & $67(41-87)$ & $67.5(32-81)$ & 0.909 \\
\hline Gender & & & 0.534 & & & 0.550 & & & 0.205 \\
\hline Female & 26 & 5 & & 28 & 0 & & 25 & 6 & \\
\hline Male & 51 & 14 & & 60 & 3 & & 56 & 6 & \\
\hline TNM stage & & & 0.383 & & & 1.000 & & & 1.000 \\
\hline $\mathrm{pTa}+\mathrm{pT} 1$ & 22 & 3 & & 22 & 1 & & 22 & 3 & \\
\hline$\geq$ pT2 & 55 & 16 & & 66 & 2 & & 59 & 9 & \\
\hline Pathology stage & & & 1.000 & & & 0.333 & & & 0.871 \\
\hline G1 & 12 & 3 & & 15 & 0 & & 14 & 1 & \\
\hline G2 & 7 & 2 & & 8 & 1 & & 8 & 1 & \\
\hline G3 & 58 & 14 & & 65 & 2 & & 59 & 10 & \\
\hline Tumor size & & & 0.244 & & & 0.571 & & & 1.000 \\
\hline$<3 \mathrm{~cm}$ & 36 & 7 & & 41 & 1 & & 37 & 5 & \\
\hline$\geq 3 \mathrm{~cm}$ & 24 & 9 & & 29 & 2 & & 27 & 4 & \\
\hline LN metastasis & & & 1.000 & & & 1.000 & & & 1.000 \\
\hline No & 70 & 18 & & 81 & 3 & & 75 & 11 & \\
\hline Yes & 7 & 1 & & 7 & 0 & & 6 & 1 & \\
\hline Distant metastasis & & & 0.488 & & & 1.000 & & & 0.343 \\
\hline No & 75 & 18 & & 85 & 3 & & 79 & 11 & \\
\hline Yes & 2 & 1 & & 3 & 0 & & 2 & 1 & \\
\hline Recurrence & & & 0.174 & & & 1.000 & & & 0.430 \\
\hline No & 75 & 17 & & 84 & 3 & & 78 & 11 & \\
\hline Yes & 2 & 2 & & 4 & 0 & & 3 & 1 & \\
\hline
\end{tabular}

LN: Lymph node; mt: mutant; wt: wild type; UC: Ureter carcinoma. $P$ values in bold indicate significant differences.

Table 4. Clinic-pathological variables and association with TERT and PLEKHSI promoter and GPR 126 intron 6 mutations in UBC patients

\begin{tabular}{|c|c|c|c|c|c|c|c|c|c|}
\hline & \multicolumn{3}{|c|}{ TERT promoter $(\mathrm{N}=106)$} & \multicolumn{3}{|c|}{ PLEKHS1 promoter $(\mathrm{N}=106)$} & \multicolumn{3}{|c|}{ GPR126 intron $6(\mathrm{~N}=102)$} \\
\hline & wt & $\mathrm{mt}$ & $P$ & wt & $\mathrm{mt}$ & $P$ & wt & $\mathrm{mt}$ & $P$ \\
\hline & $59 / 106(55.7 \%)$ & $47 / 106(44.3 \%)$ & & $78 / 106(73.6 \%)$ & $28 / 106(26.4 \%)$ & & $70 / 102(68.6 \%)$ & $32 / 102(31.4 \%)$ & \\
\hline Age (Median, years) & $66(36-87)$ & $64(44-89)$ & 0.055 & $64.5(36-86)$ & $67(43-89)$ & 0.253 & $65(36-89)$ & $64(50-87)$ & 0.751 \\
\hline Gender & & & 0.041 & & & 0.512 & & & 0.304 \\
\hline Male & 47 & 44 & & 68 & 23 & & 58 & 29 & \\
\hline Female & 12 & 3 & & 10 & 5 & & 12 & 3 & \\
\hline TNM stage & & & 0.802 & & & 0.337 & & & 0.765 \\
\hline $\mathrm{pTa}+\mathrm{pT} 1$ & 44 & 33 & & 54 & 23 & & 50 & 24 & \\
\hline$\geq \mathrm{pT} 2$ & 15 & 10 & & 20 & 5 & & 17 & 7 & \\
\hline Pathology stage & & & 0.345 & & & 0.885 & & & 1.000 \\
\hline G1 & 6 & 2 & & 6 & 2 & & 5 & 2 & \\
\hline G2 & 19 & 20 & & 30 & 9 & & 25 & 11 & \\
\hline G3 & 34 & 25 & & 42 & 17 & & 40 & 19 & \\
\hline Tumor size & & & 0.242 & & & 0.496 & & & 0.006 \\
\hline$<3 \mathrm{~cm}$ & 23 & 24 & & 36 & 11 & & 25 & 21 & \\
\hline$\geq 3 \mathrm{~cm}$ & 35 & 23 & & 41 & 17 & & 44 & 11 & \\
\hline Number of tumors & & & 0.955 & & & 1.000 & & & 0.552 \\
\hline Single & 33 & 27 & & 44 & 16 & & 41 & 17 & \\
\hline Multiple & 25 & 20 & & 33 & 12 & & 28 & 15 & \\
\hline Recurrence & & & 0.021 & & & 0.762 & & & 0.697 \\
\hline No & 52 & 33 & & 62 & 23 & & 57 & 25 & \\
\hline Yes & 7 & 14 & & 16 & 5 & & 13 & 7 & \\
\hline
\end{tabular}

LN: Lymph node; mt: mutant; wt: wild type; UBC: Urothelial bladder carcinoma. $P$ values in red indicate significant differences.

Table 5. Consistency of TERT/PLEKHSI/GPR 126 mutations between tumor tissues and urine samples from patients with UTUC and UBC as determined using Sanger sequencing

\begin{tabular}{llllll}
\hline \multirow{2}{*}{ Urine samples } & \multicolumn{3}{l}{ Tissue samples } & & \\
\cline { 2 - 5 } & MT (n) & WT (n) & Sensitivity (\%) & Specificity (\%) & Accuracy (\%) \\
\hline MT (n) & 36 & 3 & $61 \%$ & $90 \%$ & $70.7 \%$ \\
WT (n) & 23 & 27 & & & \\
Total & 59 & 30 & & & \\
\hline
\end{tabular}

MT: Mutant; WT: Wild type; UBC: Urothelial bladder carcinoma; UTUC: Upper track urothelial carcinoma.

\section{Discussion}

The recurrent TERT promoter mutations have been previously identified in UTUCs and UBCs, and the mutant DNA sequences are detectable in patient urine, thereby serving as a urinary biomarker for UTUC and UBC diagnostics [10-12, 24, 25]. Such an approach doesn't work for tumors carrying a wt TERT promoter, including most UTUC patients who lack 
TERT promoter mutations. In the present study, we comprehensively analyzed the non-coding mutations in TERT and PLEKHS1 promoters and GPR126 intron 6 in UTUCs, and demonstrated that these mutations all occurred in primary UTUC tumors.

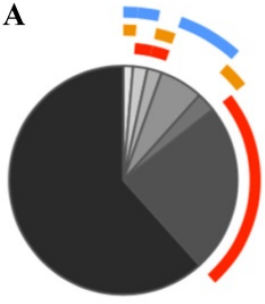

UTUC

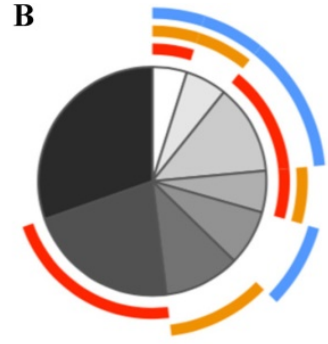

UBC

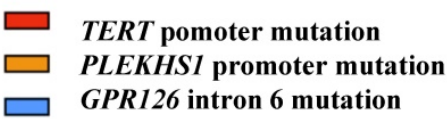

Figure 2. Proportions of different mutations in UTUC and UBC cohorts. (A) Annotated pie chart for mutation frequencies in UTUC cohort $(n=146)$. (B) Annotated pie chart for mutation frequencies in UBC cohort $(n=102)$.

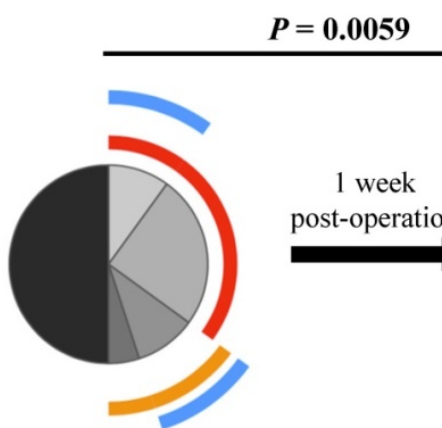

Preoperative urine

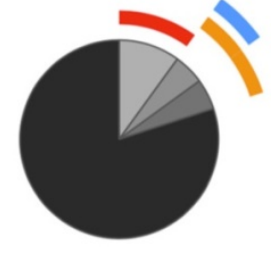

Postoperative urine

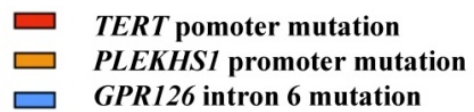

Figure 3. Differential proportions of mutated fragments detected in pre-operative and post-operative urine samples. Permutation test was used to test for significance in comparison between pre-operative and post-operative urine samples $(n=20)$.

The early detection of UTUCs is currently challenging, and it is thus more demanding to develop reliable methods for this purpose $[1,10,12]$. Urine-based tests are a non-invasive diagnostic tool for UTUC, but require specific biomarkers. Because normal urothelial cells lack mutations in the TERT and PLEKHS1 promoters or GPR126 intron 6, they should be ideal urinary biomarkers for UTUC diagnosis. Indeed, these mutant DNA sequences were found in urine from patients with mutation-bearing tumors, which indicate that these mutation assays have potentially clinical implications in UTUCs. In fact, the mutated TERT promoter has been previously evaluated as a urinary biomarker for UTUC or UBC diagnostics and disease surveillance, and the promising results have been obtained [10-12, 24, 25]. Interestingly, Hosen et al found that the TERT promoter mutation could be present in urine as early as 10 years before a clinical diagnosis of UBC [25]. Like UBCs, UTUCs also originate from the urothelium and belong to transitional cell carcinomas, and it is thus plausible that this genetic alteration occurs much earlier in UBUCs, too. Moreover, PLEKHS1 promoter and GPR126 intron 6 mutations may similarly take place in the precursor lesion during UTUC pathogenesis. If confirmed, all these three mutations will be perfect urinary biomarkers to screen the general population with UTUC/UBC risk factors.

In the present study, we determined the mutations of TERT and PLEKHS1 promoters and GPR126 intron 6 using Sanger sequencing, a gold standard for the identification of mutant targets. Based on our previous report, the threshold sensitivity of Sanger sequencing is at least $10 \%$ of mutant TERT promoter-containing tumor DNA [10]. However, voided urine contains both normal and malignant cells, and if the later fraction is too small, it might be difficult to catch the mutant target by Sanger sequencing. Such a scenario is expected with small either primary or recurring tumors producing few exfoliated tumor cells. Indeed, the present analysis results of patient urine only had less than $50 \%$ of sensitivity for Sanger sequencing. Thus, it is strongly motivated to develop more sensitive assays to detect minor proportions of mutant alleles present in bulk urinary DNA. For instance, castPCR and digital droplet PCR have been developed to analyze the mutated TERT promoter in urine derived from UTUC and UBC patients with much higher sensitivity and specificity $[10,26]$. These same techniques should also be established for PLEKHS1 promoter and GPR126 mutation assays. In addition, next generation sequencing has also emerged as a powerful tool to simultaneously analyze multiple targets including TERT and PLEKHS1 promoter and GPR126 intron 6 mutations.

The mutations of these three genes were also evaluated for their clinic-pathological association in UTUCs. Neither PLEKHS1 promoter nor GPR126 intron 6 mutations were significantly correlated with clinic-pathological variables, which was consistent with other reports on UBCs [19, 20]. Nonetheless, PLEKHS1 over-expression has been shown to predict shorter survival in UBC patients, while higher GPR126 levels seem to be associated with favourable outcomes [19, 20]. In accordance, we found that PLEKHS1 drove AKT hyperactivity, promoted metastasis and was associated with shorter survival in 
thyroid cancer [23]. For TERT promoter mutations, we observed a strongly positive correlation between the mutation and disease recurrence in UBCs. The TERT promoter mutation creates de novo ETS binding motifs to activate the TERT transcription and telomerase, thereby empowering cancer cells with a proliferation advantage [17]. Moreover, TERT displays multiple activities and is capable of protecting cancer cells from apoptosis stimulated by various insults and facilitating invasion or metastasis by inducing epithelial-mesenchymal transition or other pro-metastatic cascades [9, 27-29].

\section{Conclusions}

TERT and PLEKHS1 promoter and GPR126 intron mutations occur in UTUCs, although the frequencies are lower than those in UBC. Importantly, these mutated DNA fragments are detectable in patient urine. Because the mutations are absent in normal urothelial cells, they may serve as specific biomarkers for UTUC diagnostics and disease monitoring, which is supported by our proof-concept study. Liquid biopsies including non-invasive urinary tests for cancer diagnosis and surveillance have become more and more attractive, and toward this purpose, reliable biomarkers together with highly sensitive, specific methods such as ddPCR are required. It should also be pointed out that the mutations of TERT and PLEKHS1 promoters and GPR126 intron 6 do not occur in all patients, and these mutation assays are not applicable for patients who lack these genetic alterations. Therefore, we still need to look for new biomarkers to include remaining patients. In the meanwhile, advanced technologies including next generation sequencing should be useful by analyzing multi-targets simultaneously. Finally, the close association between TERT promoter mutations and recurrent disease may be valuable in UTUC/UBC prognostication, treatment decision and follow-up designs. Taken together, the present findings are implicated in the diagnostics and management of patients with UTUC and UBC.

\section{Abbreviations}

RPC: renal pelvic carcinoma; TERT: Telomerase reverse transcriptase; UBC: urothelial bladder carcinoma; UC: Ureter carcinoma; UTUC: upper track urothelial carcinoma.

\section{Acknowledgments}

\section{Funding}

This work was supported by grants from the Swedish Cancer Society No. 190018 Pj), Swedish Research Council (2018-02993), the Cancer Society in
Stockholm (171223), Stockholm County Council and Karolinska Institutet (2018-01524), Lars Hiertas Foundation, Swedish Foundation for International Cooperation in Research and Higher Education (STINT, CH2016-6632), National Natural Science Foundation of China (No. 81711530048 and 81572515) and Young Scholars Program of Shandong University. $X Y$ was supported by the China Postdoctoral Science Foundation Grant (2019M652404 and 2020T130372).

\section{Authors' contributions}

$X X, T L, M B$ and $D X$ contributed to the conceptualization of the study. XX, XY, TL and MD were responsible for the methodology design. $X X, T L$, $\mathrm{YF}, \mathrm{CL}$, and KS conducted to verify the experimental results and to interpret the data. XX, TL and DX performed writing original draft preparation. KS, YF, $\mathrm{CL}$, and $\mathrm{MB}$ were involved in writing-review \& editing. TL, MB and DX provided supervision and funding acquisition. All authors read and approved the final manuscript.

\section{Availability of data and materials}

The datasets used and/or analyzed during the current study are available from the corresponding author on reasonable request.

\section{Competing Interests}

The authors have declared that no competing interest exists.

\section{References}

1. Szarvas T, Modos O, Horvath A, Nyirady P. Why are upper tract urothelial carcinoma two different diseases? Transl Androl Urol. 2016, 5(5):636-647.

2. Chen $\mathrm{W}$, Zheng R, Baade PD, Zhang S, Zeng H, Bray F, et al. Cancer statistics in China, 2015. CA Cancer J Clin. 2016, 66(2):115-132.

3. Chromecki TF, Bensalah K, Remzi M, Verhoest G, Cha EK, Scherr DS, et al. Prognostic factors for upper urinary tract urothelial carcinoma. Nat Rev Urol. 2011, 8(8):440-447.

4. Baard J, de Bruin DM, Zondervan PJ, Kamphuis G, de la Rosette J, Laguna MP. Diagnostic dilemmas in patients with upper tract urothelial carcinoma. Nat Rev Urol. 2017, 14(3):181-191.

5. Soria F, Shariat SF, Lerner SP, Fritsche HM, Rink M, Kassouf W, et al. Epidemiology, diagnosis, preoperative evaluation and prognostic assessment of upper-tract urothelial carcinoma (UTUC). World J Urol. 2017, 35(3):379-387.

6. Kang M, Yoo H, Kim K, Sung SH, Jeon HG, Park SH, et al. Role of Adjuvant Chemotherapy in Advanced Stage Upper Urinary Tract Urothelial Carcinoma after Radical Nephroureterectomy: Competing Risk Analysis after Propensity Score-Matching. J Cancer. 2019, 10(27):6896-6902.

7. Wang LJ, Chou WC, Pang ST, Yang CW, Chuang CK, Chang YH, et al. Risk Stratification of Upper Urinary Tract Urothelial Carcinoma Patients for Survival Prediction: A Simple Summation Scoring Method. J Cancer. 2018, 9(13):2284-2294.

8. Yuan X, Dai M, Xu D. Telomere- Related Markers for Cancer. Curr Top Med Chem. 2020, 20(6):410 - 432

9. Yuan $\mathrm{X}$, Larsson $\mathrm{C}, \mathrm{Xu}$ D. Mechanisms underlying the activation of TERT transcription and telomerase activity in human cancer: old actors and new players. Oncogene. 2019, 38(34):6172-6183.

10. Wang K, Liu T, Ge N, Liu L, Yuan X, Liu J, et al. TERT promoter mutations are associated with distant metastases in upper tract urothelial carcinomas and serve as urinary biomarkers detected by a sensitive castPCR. Oncotarget. 2014, 5(23):12428-12439.

11. Wang K, Liu T, Liu C, Meng Y, Yuan X, Liu L, et al. TERT promoter mutations and TERT mRNA but not FGFR3 mutations are urinary biomarkers in Han Chinese patients with urothelial bladder cancer. Oncologist. 2015, 20(3):263-269. 
12. Wang K, Liu T, Liu L, Liu J, Liu C, Wang C, et al. TERT promoter mutations in renal cell carcinomas and upper tract urothelial carcinomas. Oncotarget. 2014, 5(7):1829-1836.

13. Guo Y, Yuan $\mathrm{X}, \mathrm{Li} \mathrm{K}$, Dai M, Zhang $\mathrm{L}, \mathrm{Wu} \mathrm{Y}$, et al. GABPA is a master regulator of luminal identity and restrains aggressive diseases in bladder cancer. Cell Death Differ. 2020, 27(6):1862-1877.

14. Liu X, Wu G, Shan Y, Hartmann C, von Deimling A, Xing M. Highly prevalent TERT promoter mutations in bladder cancer and glioblastoma. Cell Cycle. 2013, 12(10):1637-1638

15. Giedl J, Rogler A, Wild A, Riener MO, Filbeck T, Burger M, et al. TERT Core Promotor Mutations in Early-Onset Bladder Cancer. J Cancer. 2016, 7(8):915-920.

16. Bell RJ, Rube HT, Kreig A, Mancini A, Fouse SD, Nagarajan RP, et al. The transcription factor GABP selectively binds and activates the mutant TERT promoter in cancer. Science. 2015, 348(6238):1036-1039.

17. Yuan $X$, Dai M, Xu D. TERT promoter mutations and GABP transcription factors in carcinogenesis: More foes than friends. Cancer Lett. 2020, 493:1-9.

18. Weinhold N, Jacobsen A, Schultz N, Sander C, Lee W. Genome-wide analysis of noncoding regulatory mutations in cancer. Nat Genet. 2014, 46(11):1160-1165.

19. Garinet S, Pignot G, Vacher S, Le Goux C, Schnitzler A, Chemlali W, et al High Prevalence of a Hotspot of Noncoding Somatic Mutations in Intron 6 of GPR126 in Bladder Cancer. Mol Cancer Res. 2019, 17(2):469-475.

20. Pignot G, Le Goux C, Vacher S, Schnitzler A, Radvanyi F, Allory Y, et al. PLEKHS1: A new molecular marker predicting risk of progression of non-muscle-invasive bladder cancer. Oncol Lett. 2019, 18(4):3471-3480.

21. Lopez-Beltran A, Scarpelli M, Montironi R, Kirkali Z. 2004 WHO classification of the renal tumors of the adults. Eur Urol. 2006, 49(5):798-805.

22. Liu T, Wang N, Cao J, Sofiadis A, Dinets A, Zedenius J, et al. The age- and shorter telomere-dependent TERT promoter mutation in follicular thyroid cell-derived carcinomas. Oncogene. 2014, 33(42):4978-4984.

23. Xing $\mathrm{X}, \mathrm{Mu} \mathrm{N}$, Yuan $\mathrm{X}$, Wang $\mathrm{N}$, Juhlin CC, Straat $\mathrm{K}$, et al. PLEKHS1 Over-Expression is Associated with Metastases and Poor Outcomes in Papillary Thyroid Carcinoma. Cancers. 2020, 12(8):2133.

24. Hurst CD, Platt FM, Knowles MA. Comprehensive mutation analysis of the TERT promoter in bladder cancer and detection of mutations in voided urine. Eur Urol. 2014, 65(2):367-369.

25. Hosen MI, Sheikh M, Zvereva M, Scelo G, Forey N, Durand G, et al. Urinary TERT promoter mutations are detectable up to 10 years prior to clinical diagnosis of bladder cancer: Evidence from the Golestan Cohort Study. EBioMedicine. 2020, 53:102643.

26. Hosen MI, Forey N, Durand G, Voegele C, Bilici S, Avogbe PH, et al. Development of Sensitive Droplet Digital PCR Assays for Detecting Urinary TERT Promoter Mutations as Non-Invasive Biomarkers for Detection of Urothelial Cancer. Cancers. 2020, 12(12):3541.

27. Liu Z, Li Q, Li K, Chen L, Li W, Hou M, Liu T, et al. Telomerase reverse transcriptase promotes epithelial-mesenchymal transition and stem cell-like traits in cancer cells. Oncogene. 2013, 32(36):4203-4213.

28. Ding D, Xi P, Zhou J, Wang M, Cong YS. Human telomerase reverse transcriptase regulates MMP expression independently of telomerase activity via NF-kappaB-dependent transcription. FASEB J. 2013, 27(11):4375-4383.

29. Yu J, Yuan X, Sjoholm L, Liu T, Kong F, Ekstrom TJ, et al. Telomerase reverse transcriptase regulates DNMT3B expression/aberrant DNA methylation phenotype and AKT activation in hepatocellular carcinoma. Cancer Lett. 2018, 434:33-41 